\title{
Desarrollo y aplicación de contenidos educativos digitales desde un teléfono inteligente para un tema de Estadística en un curso
} universitario

Development and application of educational digital contents for their access in smartphones about a statistic topic of a university course

\section{Volumen 19, Número 1}

Enero-Abril

pp. 1-22

Este número se publica el 1 de enero de 2019

DOI 10.15517/aie.v19i1.35711

Javier Organista-Sandoval

Claudia Domínguez Pérez

Maricela López Ornelas

Revista indizada en REDALYC, $\underline{\text { SCIELO }}$

Revista distribuida en las bases de datos:

LATINDEX, DOAJ, REDIB, IRESIE, CLASE, DIALNET, SHERPA/ROMEO, QUALIS-CAPES, MIAR

Revista registrada en los directorios:

ULRICH'S, REDIE, RINACE, OEI, MAESTROTECA, PREAL, CLACSO 


\title{
Desarrollo y aplicación de contenidos educativos digitales desde un teléfono inteligente para un tema de Estadística en un curso universitario
}

\author{
Development and application of educational digital contents for their access in smartphones \\ about a statistic topic of a university course
}

\author{
Javier Organista-Sandoval ${ }^{1}$ \\ Claudia Domínguez Pérez² \\ Maricela López Ornelas ${ }^{3}$
}

\begin{abstract}
Resumen: El propósito del artículo es describir el desarrollo de contenidos educativos digitales para un tema estadístico y su consulta desde un teléfono inteligente. La aplicación de los contenidos se realizó en el contexto de un curso formal universitario y permitió recuperar información del porcentaje de posesión de teléfonos inteligentes del grupo participante. De la misma forma, se obtuvieron datos relacionados con los aspectos tecnológicos y de diseño, del contexto de la consulta, del agrado y desagrado de los propios desarrollos. Se expusieron tres temas estadísticos basados en el diseño instruccional ADDIE (Análisis, Diseño, Desarrollo, Implementación y Evaluación). La selección de la muestra intencional consideró 40 estudiantes universitarios, a quienes se les aplicó un cuestionario con el propósito de identificar las bondades y limitaciones de este tipo de desarrollo. Los resultados mostraron que todo el estudiantado dispone de un teléfono inteligente con conexión a Internet, por lo que fue posible acceder de forma ubicua y anticipada a los contenidos. Asimismo, se señalaron aspectos favorables al diseño, como la organización, funcionalidad y utilidad de los contenidos educativos consultados. Las principales limitaciones fueron las explicaciones concretas y la corta duración de los segmentos audiovisuales. Los distractores identificados hacen mención del ruido ambiental, las notificaciones de redes sociales y mensajes de texto. Los hallazgos sugieren que el teléfono inteligente puede funcionar como una herramienta pedagógica con una adecuada orientación en su uso.
\end{abstract}

Palabras clave: Aprendizaje móvil, innovación pedagógica, técnica pedagógica

\begin{abstract}
This article describes the development of digital educational contents for a statistic topic that can be consulted in a smartphone. Frequently, this device is restricted in learning spaces because it is a distractor; consequently, in this article it is intended to explore the educational use of the device that can access to educational contents in a ubiquitous way. For that purpose, three topics in statistic has been developed based in the instructional design ADDIE (Analysis, Design, Development, Implementation and Evaluation). The contents were implemented in a formal course of university. The opinion of 40 students was recovered through a questionnaire with the purpose of identify the benefits and limitations in this kind of development. The results revealed that all the students have smartphones with Internet connection; this allowed them the access to that content in ubiquitous way and in advance. Among the benefits are the organization, design, functionality and utility of the consulted educational materials. The major limitations were brief explanations and limited duration in the audiovisual segments. The identified distractors were environmental noise, social media notifications and text messages. The described experience shows that the smartphone device can function as an educational tool if it is lead correctly.
\end{abstract}

Keywords: Mlearning, Educational methods, teaching techniques

\footnotetext{
${ }^{1}$ Instituto de Investigación y Desarrollo Educativo (IIDE), Universidad Autónoma de Baja

California (UABC), México. Dirección electrónica: javor@uabc.edu.mx

2 Instituto de Investigación y Desarrollo Educativo (IIDE) de la Universidad Autónoma de

Baja California (UABC), México. Dirección electrónica: domin-quez.claudia@uabc.edu.mx

${ }^{3}$ Instituto de Investigación y Desarrollo Educativo (IIDE), Universidad Autónoma de Baja

California (UABC), México. Dirección electrónica: ornelas@uabc.edu.mx
}

Artículo recibido: 06 de junio, 2018

Enviado a corrección: 08 de setiembre, 2018

Aprobado: 10 de diciembre, 2018 


\section{Introducción}

La sociedad de la ubicuidad —otro de los diversos nombres adjudicados a la sociedad del siglo XXI—se distingue por su frecuente exposición al llamado ecosistema de medios. Las actividades diarias transcurren bajo una frecuente exposición a distintas tecnologías y a diversos medios de información y comunicación. La existencia de sus integrantes transcurre, de forma típica, con la consulta al teléfono celular, la ejecución de aplicaciones de comunicación como Facebook o WhatsApp, el encendido de la computadora portátil, por mencionar algunos ejemplos.

En el caso del segmento poblacional joven, y particularmente del estudiantado universitario, según la Encuesta Nacional sobre Disponibilidad y Uso de Tecnologías de la Información en los Hogares del Instituto Nacional de Estadística y Geografía (INEGI) (2018), un porcentaje elevado del estudiantado de nivel superior poseeun teléfono celular (92\%) y dispone de conexión a Internet (95\%). Los valores expresados, sugieren una tendencia a estar conectado a algún objeto tecnológico que le proporcione información o acceso a una red social. En correspondencia, dentro del contexto tecnológico, prácticamente cualquier persona, en cualquier momento y lugar, puede consumir o producir información de Internet. Es decir, las características de conectividad y ubicuidad de los estudiantes, permite visualizar los teléfonos inteligentes, como un dispositivo susceptible de utilizarse como herramienta de apoyo en las actividades educativas.

Esta creciente exposición tecnológica, de acuerdo con Aparici (2011), genera retos y conflictos, los cuales surgen por el hecho de que la mayoría de las escuelas realizan sus actividades educativas desde una didáctica tradicional; sin embargo, fuera del aula, el estudiante pone en práctica modelos basados en la colaboración, la interacción, el acceso a los recursos mediáticos -entre otros- como forma de apoyar sus actividades extraescolares. El reto es precisamenterepensar un modelo educativo donde la mediación tecnológica sea percibida como un elemento de apoyo en las actividades pedagógicas y administrativas.

De forma tal que la posibilidad de acceder a información académica por la vía de medios electrónicos se considere como una ventana de oportunidades para la administración de contenidos educativos. Por consiguiente, el propósito del documento, es describir el desarrollo y aplicación de contenidos educativos digitales para una temática específica de un curso universitario de Estadística básica, con una orientación en el diseño instruccional para 
ser consultada desde un teléfono inteligente. Los contenidos educativos generados fueron administrados en un curso formal universitario, lo que permitió recuperar información pedagógica y tecnológica en torno a la experiencia. Además, se señalan algunas bondades y limitaciones del tipo de intervención a partir de la opinión del estudiantado.

\section{Referentes}

El teléfono inteligente, también conocido como smartphone, es un dispositivo portátil que ha ganado popularidad en la comunidad estudiantil de nivel superior en México (Instituto Nacional de Estadística y Geografía, 2018; Organista-Sandoval y Serrano-Santoyo, 2014). La información estadística reportada sostiene que tres de cada cuatro usuarios de telefonía celular cuentan con uno, y su uso se orienta mayoritariamente (88.9\%) a establecer comunicación vía Internet y/o acceder a contenidos digitales.

Las características más relevantes de los teléfonos inteligentes son su portabilidad y conectividad, que le conceden al usuario acceso en todo momento y lugar a información y a procesos de comunicación por diferentes vías. Las oportunidades pedagógicas que pueden apoyarse con tales características han motivado el interés de la comunidad estudiantil, especialmente del nivel educativo superior, por lo que dicho dispositivo ya es considerado como una herramienta pedagógica básica. Como referente, en el contexto estudiantil donde se desarrolló el presente estudio, el nivel de posesión de teléfonos inteligentes es de 91\% (Organista-Sandoval, Sandoval, McAnally y Lavigne, 2016). Este porcentaje coincide con el reportado por Lagunes-Domínguez, Torres-Gastelú, Angulo-Armenta y Martínez-Olea (2017), en cuya investigación incluyeron estudiantes del nivel superior de México y Colombia, señalando una cobertura casi total de dispositivos portátiles de la comunidad de estudio y la disposición para su manejo en actividades de aprendizaje móvil.

Si bien, se ha señalado que el teléfono inteligente puede funcionar como una herramienta de apoyo al proceso educativo, también consigue operar como un distractor en el aula (Landín, Francisco y Santiago, 2015), en gran medida, por el acceso ubicuo a una amplia gama de aplicaciones de entretenimiento, redes sociales, mensajería instantánea, videos, música, entre otros. Lo anterior ha propiciado que en algunos centros educativos se restrinja o prohíba su uso dentro del aula. De acuerdo con Chiappe (2016), ello no coincide con la postura de la Organización de las Naciones Unidas para la Educación, la Ciencia y la Cultura (UNESCO), ya que dicho organismo expresa la importancia de fomentar el uso de la 
tecnología con propósitos educativos y el reconocimiento de los dispositivos portátiles como herramientas pedagógicas con posibilidades de apoyar las actividades escolares.

Para Díaz, Saucedo, Salinas y Jiménez (2014), el alumnado tiene un papel fundamental para cambiar las prácticas que limitan o prohíban el uso de dispositivos portátiles dentro del aula, ya que, precisamente, es la comunidad estudiantil quien puede contribuir a la aceptación responsable de su uso. De esta manera, puede evitarse o disminuir el rol distractor de tales dispositivos. Indudablemente se requiere de una labor de orientación y capacitación para lograr el uso apropiado de los recursos tecnológicos en las actividades educativas. Una posibilidad de explotar el potencial pedagógico de los teléfonos inteligentes es proporcionarle contenidos educativos digitales al alumnado para su consulta en los diversos dispositivos, independientemente del tiempo y del lugar. Donkor (2011) menciona que una de las ventajas del desarrollo de estos materiales educativos es que le permite al estudiantado visualizar varias veces el contenido, detener o regresar al punto específico de la lección, o donde se requiera más tiempo de comprensión.

A nivel internacional, se desarrolló una experiencia interesante de apoyo a las actividades educativas por varias organizaciones, empresas y universidades, entre las que se encuentran: UNESCO, la compañía Apple INC, la compañía MyON (Servicios de Literacidad personalizada), Universidad de las Américas en Puebla, la Universidad de Cambridge (Cambridge English Language Assessment), la organización ETS (Educational Testing Service), creadora del examen TOEFL, entre otras. A través del sistema llamado UNOi, alojado en el sitio web http://mx.unoi.com, brindan herramientas innovadoras mediante materiales para su consulta desde un dispositivo iPad. Dicho sistema opera en una red de más de mil colegios, principalmente en Ecuador, Colombia, Brasil y México. Su aporte se basa en visualizar la tecnología como una herramienta pedagógica de acompañamiento a la persona estudiante, quien recurre a ella como una persona creativa e innovadora.

Sin embargo, para lograr ofrecer contenidos educativos digitales para su consulta desde un teléfono inteligente se requiere de un diseño instruccional que considere las particularidades técnicas de tales dispositivos, como pantalla de tamaño reducido y aplicaciones con bajo consumo de recursos, por mencionar algunas. La idea, tras un diseño instruccional, es organizar la instrucción en fases basadas en teorías del aprendizaje, que sustenten la meta pedagógica propuesta (Chiappe, 2008; Agudelo, 2009). 
Una investigación relevante en torno a un desarrollo de mlearning fue realizada por Otero (2014), quien propuso un modelo de aprendizaje móvil dirigido al nivel educativo superior (OpenEducaML). Dicho modelo se fundamenta en la teoría del constructivismo social, el aprendizaje colaborativo, conectivista, abierto y distribuido. La autora señala la inexistencia de una teoría del aprendizaje orientada al aprendizaje móvil y la complejidad de su modelado. A su vez, destaca el potencial pedagógico de las herramientas móviles en el nivel superior. Por su parte, Denham, Quick y Atkinson (2012) coinciden en la importancia de la movilidad y el contexto para favorecer al aprendizaje. No obstante, sostienen que las posturas epistemológicas del aprendizaje móvil no son una reformulación de aquellas que provienen del elearning o de las teorías clásicas.

De acuerdo con Mancinas (2018), el aprendizaje móvil favorece una cognición de tipo empírico, basada en la búsqueda de la solución a un problema, a partir de datos y su experimentación entre lo algorítmico y lo heurístico. Para dicho autor, el teléfono inteligente le demanda al estudiante una actitud activa, de exploración y experimentación, que contrasta con la actitud pasiva del estudiante espectador en el aula. El aprendizaje móvil confluye con una amplia gama de factores de influencia que dificultan su caracterización desde una postura epistemológica única. Dicho autor destaca las prácticas de aprendizaje con uso de recursos tecnológicos, la colaboración en las redes sociales y el acceso al conocimiento distribuido en Internet. Coincide con Denham, Quick y Atkinson (2012) acerca del interés que muestra la comunidad estudiantil hacia el uso del teléfono inteligente como soporte para el aprendizaje

El interés hacia el uso de recursos tecnológicos portátiles en educación, como objeto de investigación, queda de manifiesto en la Tabla 1, donde se presentan algunos trabajos afines con la temática de este documento. 


\section{Tabla 1}

Investigaciones relacionadas con el uso de dispositivos portátiles en educación

\begin{tabular}{|c|c|c|c|}
\hline Autor & Desarrollo & Diseño Instruccional & Contexto \\
\hline $\begin{array}{l}\text { Mancinas } \\
(2018)\end{array}$ & $\begin{array}{l}\text { Uso de dispositivos } \\
\text { móviles en un curso } \\
\text { universitario. Interés en } \\
\text { identificar el tipo de } \\
\text { cognición. }\end{array}$ & $\begin{array}{l}\text { Se destaca la cognición de tipo } \\
\text { empírico; aprendizaje basado en } \\
\text { problemas; esquema de } \\
\text { experimentación con enfoque } \\
\text { algorítmico y heurístico. }\end{array}$ & $\begin{array}{l}\text { México } \\
\text { Estudiantes de } \\
\text { educación } \\
\text { superior. }\end{array}$ \\
\hline $\begin{array}{l}\text { Lagunes- } \\
\text { Dominguez, et } \\
\text { al. (2018) }\end{array}$ & $\begin{array}{l}\text { Estudio comparativo con } \\
\text { estudiantes colombianos y } \\
\text { mexicanos. }\end{array}$ & $\begin{array}{l}\text { Se orienta a recuperar } \\
\text { información sobre posesión de } \\
\text { dispositivos portátiles y } \\
\text { disposición para el aprendizaje } \\
\text { móvil }\end{array}$ & $\begin{array}{l}\text { Colombia y } \\
\text { México. } \\
\text { Estudiantes de } \\
\text { educación } \\
\text { superior }\end{array}$ \\
\hline $\begin{array}{l}\text { Jeong y Cha } \\
\text { (2017) }\end{array}$ & $\begin{array}{l}\text { Aplicación para teléfonos } \\
\text { inteligentes }\end{array}$ & $\begin{array}{l}\text { Modelo de diseño instruccional de } \\
\text { Dick y Carey }\end{array}$ & $\begin{array}{l}\text { Corea del Sur. } \\
\text { Estudiantes } \\
\text { adolescentes. }\end{array}$ \\
\hline $\begin{array}{l}\text { Enrico, } \\
\text { Casanova, } \\
\text { Enrico y } \\
\text { Bossolasco } \\
(2017)\end{array}$ & $\begin{array}{l}\text { Materiales educativos } \\
\text { digitales a través de código } \\
\text { QR para dispositivos } \\
\text { portátiles }\end{array}$ & $\begin{array}{l}\text { Diseño instruccional situado. } \\
\text { Exposición a materiales } \\
\text { educativos multimedia, con uso } \\
\text { de código QR y realidad } \\
\text { aumentada }\end{array}$ & $\begin{array}{l}\text { Argentina. } \\
\text { Estudiantes de } \\
\text { educación } \\
\text { superior. }\end{array}$ \\
\hline $\begin{array}{l}\text { Velarde, } \\
\text { Dehesa, López } \\
\text { y Márquez } \\
\text { (2017) }\end{array}$ & Videos tutoriales & $\begin{array}{l}\text { Modelo de diseño instruccional de } \\
\text { Gagné }\end{array}$ & $\begin{array}{l}\text { México } \\
\text { Estudiantes de } \\
\text { educación } \\
\text { superior. }\end{array}$ \\
\hline $\begin{array}{l}\text { Basantes, } \\
\text { Naranjo, } \\
\text { Gallegos y } \\
\text { Benítez (2017) }\end{array}$ & $\begin{array}{l}\text { Objetos de Aprendizaje } \\
\text { Móvil para tabletas y } \\
\text { teléfonos inteligentes }\end{array}$ & $\begin{array}{l}\text { Diseño instruccional con } \\
\text { incorporación de elementos } \\
\text { interactivos, colaborativos y } \\
\text { actividades lúdicas }\end{array}$ & $\begin{array}{l}\text { Ecuador } \\
\text { Estudiantes de } \\
\text { educación } \\
\text { superior. }\end{array}$ \\
\hline Rosero (2015) & $\begin{array}{l}\text { Aplicación móvil para } \\
\text { tabletas y teléfonos } \\
\text { inteligentes }\end{array}$ & Diseño instruccional ADDIE & $\begin{array}{l}\text { Colombia } \\
\text { Estudiantes de } \\
\text { educación } \\
\text { superior. }\end{array}$ \\
\hline $\begin{array}{l}\text { Martínez y } \\
\text { Chávez (2015) }\end{array}$ & $\begin{array}{l}\text { Plataforma web responsiva } \\
\text { para tabletas y teléfonos } \\
\text { inteligentes }\end{array}$ & $\begin{array}{l}\text { Diseño instruccional con } \\
\text { enfoques al trabajo colaborativo, } \\
\text { aprendizaje basado en } \\
\text { problemas, interacción con } \\
\text { recursos multimedia. }\end{array}$ & $\begin{array}{l}\text { México } \\
\text { Estudiantes de } \\
\text { posgrado virtual. }\end{array}$ \\
\hline Otero (2014). & $\begin{array}{l}\text { Modelo de aprendizaje } \\
\text { móvil (OpenEducaML) }\end{array}$ & $\begin{array}{l}\text { Basado en aportaciones teóricas } \\
\text { del constructivismo social, el } \\
\text { aprendizaje colaborativo, } \\
\text { conectivista, abierto y distribuido. }\end{array}$ & $\begin{array}{l}\text { México } \\
\text { Estudiantes de } \\
\text { educación } \\
\text { superior. }\end{array}$ \\
\hline
\end{tabular}

Fuente: Elaboración propia del autor (2018)

En el contexto universitario, el mapa curricular de una carrera se articula sobre una serie de cursos que dan soporte a la meta formativa de la carrera, por lo que, la posibilidad de ofrecer contenidos digitales también es amplia. De manera específica, la presente 
investigación optó por una temática de un curso de Estadística básica al ser una materia que se encuentra presente en gran cantidad de carreras universitarias y que, como señalan Faustino y Pérez (2014), se le asocian dificultades para su enseñanza. De acuerdo con Batanero y Díaz (2004), la llamada ciencia de los datos, como se refiere a la Estadística, reviste especial importancia para la comunidad académica por la necesidad de manejo, integración e interpretación de una gran cantidad de datos que se derivan del uso de las Tecnologías de la Información y Comunicación (TIC).

Con base en lo anterior, la investigación expone un caso de estudio del desarrollo y aplicación de un contenido educativo digital de apoyo a la enseñanza de un módulo de Estadística de un curso formal universitario para su consulta desde un teléfono inteligente. La idea es anticipar los contenidos educativos a la sesión presencial del curso y aprovechar la posibilidad de revisarlos en cualquier tiempo y lugar, como estrategia de apoyo pedagógico para facilitar la comprensión de determinado tema estadístico.

\section{Método}

Se consideró un enfoque metodológico mixto, ya que se incorporan elementos cuantitativos y cualitativos en la investigación. Se recurre a la perspectiva denominada Investigación y Desarrollo $(\mathrm{I}+\mathrm{D})$, que considera un desarrollo o implementación tecnológico cuya aplicación se da en un contexto natural. La organización de las dos etapas reconoce la recuperación de información tanto del desarrollo como de su aplicación, lo que incrementa la riqueza del estudio. A continuación, se describen ambas etapas.

\subsection{Desarrollo de los Contenidos Educativos Digitales (CED)}

El desarrollo de los CED se basó en la estrategia de diseño instruccional denominada $\mathrm{ADDIE}^{2}$. El propósito fue elaborar contenidos en formato digital de apoyo a una temática de un curso de Estadística para su consulta desde un teléfono inteligente (smartphone). La primera etapa del i) análisis requirió de la opinión del docente de la materia quien sugirió el tema de contrastes no-paramétricos (Wilcoxon, U-Mann Whitney y Kruskal Wallis), dada la dificultad identificada en cursos anteriores. La organización de los contenidos incluyó la presentación del tema, conceptos básicos, problemas resueltos y conclusión. Se revisaron y

\footnotetext{
${ }^{2}$ Acrónimo de sus fases: Análisis, Diseño, Desarrollo, Implementación y Evaluación.
} 
seleccionaron los contenidos susceptibles de incorporar elementos gráficos o audiovisuales para ofrecer materiales atractivos y dinámicos (Berney y Betrancourt, 2016; Betrancourt, 2005; Lowe, 2003; Mayer, 2014).

En la segunda etapa del ii) diseño se propuso la estructura base para los CED a partir de la información proporcionada por el docente, como se muestra en la Figura 1.

Figura 1.

Estructura interna de los contenidos educativos digitales

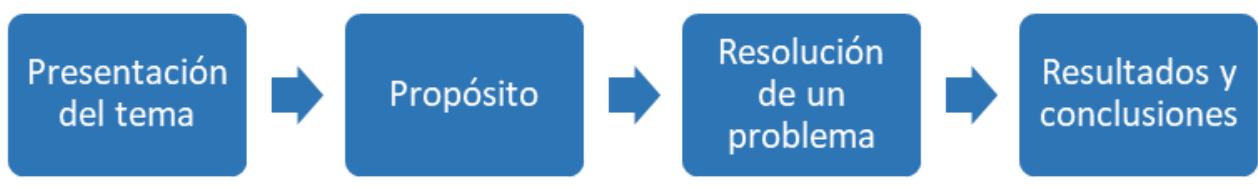

Fuente: Elaboración propia (2018).

A partir de los contenidos se elaboró el guion auditivo (ver Tabla 2), como apoyo a la realización de los guiones gráficos (storyboards), complementado con imágenes encontradas en Google.

Tabla 2.

Guion de audio donde se enuncia el problema asociado al contraste Wilcoxon

\begin{tabular}{|c|l|c|}
\hline Escena & \multicolumn{1}{|c|}{ Voz } & Duración \\
\hline$\ldots$ & $\ldots$ & $\ldots$ \\
\hline $\mathbf{4}$ & $\begin{array}{l}\text { Resolvamos un caso } \\
\text {... }\end{array}$ & $00: 03$ \\
\hline $\mathbf{6}$ & $\begin{array}{l}\text { Se desea evaluar a un docente de } \\
\text { reciente contratación. }\end{array}$ & $00: 04$ \\
\hline & $\begin{array}{l}\text { Para ello, se pide a tres grupos de } \\
\text { estudiantes que evalúen al docente con } \\
\text { una escala que va del 1 al 5, donde 1 es } \\
\text { igual a pésimo y 5 a excelente. }\end{array}$ & \\
\hline & & \\
\hline
\end{tabular}

Fuente: Elaboración propia (2018). 
El objetivo de los guiones gráficos fue proporcionar una guía visual en el desarrollo de Ios CED (ver Figura 2). La selección de colores se basó en las características típicas de iluminación de los dispositivos portátiles y el reflejo de la luz externa sobre la pantalla del dispositivo. Por ello, se utilizaron colores oscuros con texto blanco o fondos blancos con texto oscuro para favorecer el contraste. El tipo de fuente óptimo para el texto, fue Microsoft Himalaya, por su claridad y diseño ligero.

Figura 2.

Guion gráfico considerado para la elaboración del tema del contraste Wilcoxon.

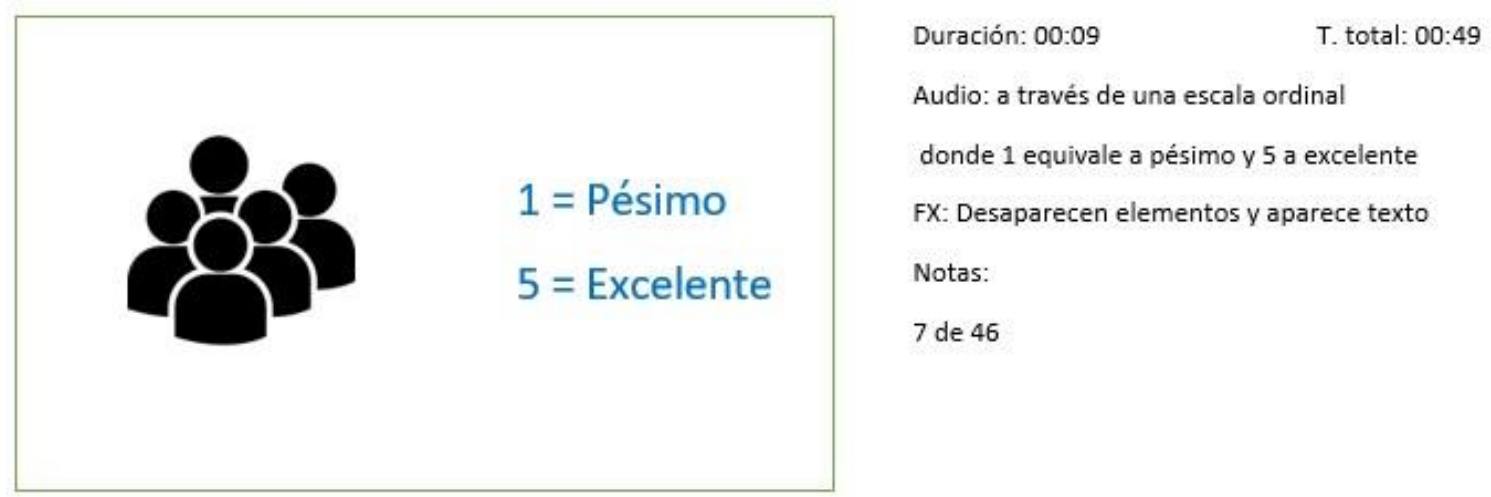

Fuente: Elaboración propia (2018).

La etapa del iii) desarrollo consistió en la elaboración de un segmento audiovisual en formato digital para su consulta desde un teléfono inteligente a través de los programas de: Adobe Illustrator, Photoshop y Creative Cloud y After Effects. La Figura 3 muestra el diseño final de la escena expuesta en la Figura 2. 
Figura 3.

Diseño de una escena para explicar el tema de contraste Wilcoxon

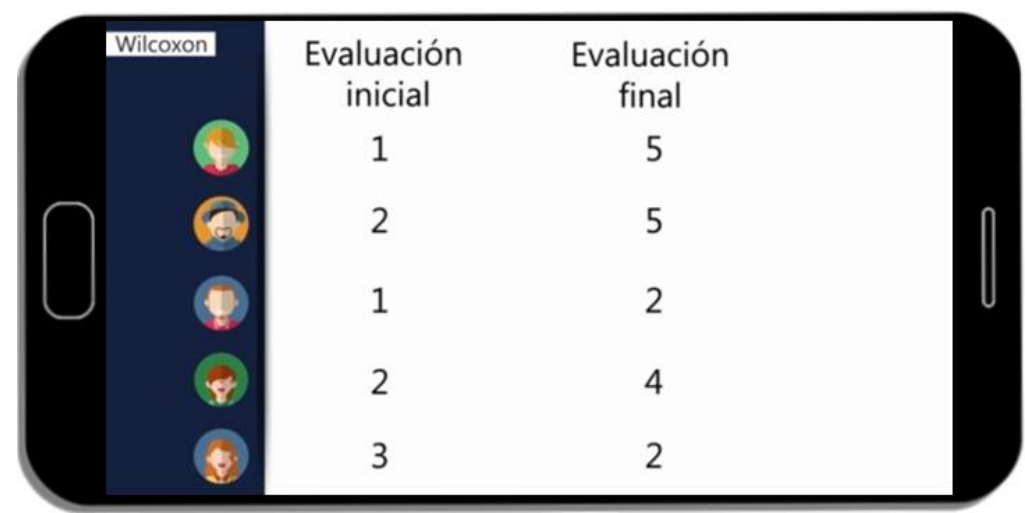

Fuente: Elaboración propia (2018).

Para la grabación y edición de audio se utilizó Adobe Audition, lo que permitió la modulación del volumen, recortar y unir segmentos de audio y la eliminación de ruido ambiental. El programa de edición audiovisual Adobe Premier Pro facilitó la integración de animación y audio para visualizarlo en tiempo real. Por tanto, se recurrió el ajuste preestablecido del programa Adobe Media Encoder para videos de alta calidad para la plataforma YouTube con formato H.264. Además, como parte de la estrategia de difusión de los contenidos educativos generados, se elaboró una página web responsiva en la plataforma Wix (http://wix.com).

La etapa de iv) implementación inicia con la realización de un pilotaje con 17 estudiantes de Maestría en Ciencias Educativas, quienes cursaban la materia de Estadística. Se les proporcionaron los CED para su consulta desde sus respectivos teléfonos inteligentes y posteriormente se solicitó su opinión en torno a la experiencia. Como parte de la estrategia de aula invertida (García- Barrera, 2013; Martínez-Olvera, Esquivel-Gámez y Martínez, 2014), la distribución de los contenidos digitales fue previa a la clase presencial, donde se utilizaron las vías de Google Classroom y WhatsApp. En cuanto a la v) evaluación de los CED, se requirió la opinión al grupo de estudiantes, en formato de preguntas abiertas, con respecto a la claridad de los contenidos, dificultades para su consulta, tipo de dispositivo utilizado y aspectos agradables y desagradables de la experiencia.

Posterior a la aplicación de las preguntas, se les pidió a los y a estudiantes resolvieran un ejercicio afín con apoyo del CED proporcionado (ver figura 4). Finalmente, se les volvió a 
preguntar acerca de los problemas que tuvieron. La información obtenida, permitió adecuar y mejorar algunos procedimientos asociados.

Figura 4.

Aplicación de los contenidos educativos digitales por parte de una estudiante universitaria

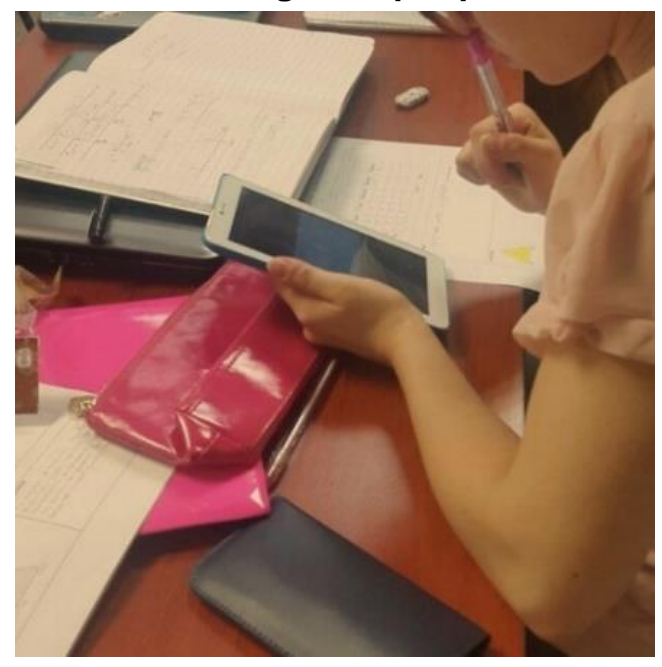

Fuente: Elaboración propia del autor (2018).

\subsection{Aplicación de los Contenidos Educativos Digitales en un contexto universitario}

Se seleccionaron de forma intencional - a conveniencia-, 40 estudiantes de tercer semestre de la licenciatura en Psicología, quienes cursaban la materia de Estadística inferencial durante el semestre agosto-diciembre de 2017. El interés por la selección particular del grupo de estudiantes recae en brindar apoyo audiovisual a una de las materias con mayor grado de dificultad en el área de humanidades y ciencias de la conducta (Batanero, 2001; Zapata, 2011).

El cuestionario utilizado tuvo el propósito de recuperar información en torno a la opinión del alumnado participante acerca de:

i) Aspectos personales. Se dirige a recuperar información del estudiante, principalmente: género, historial académico, agrado por la Estadística y sus conocimientos sobre dicha materia.

ii) Aspectos tecnológicos. Recupera información sobre la posesión, uso, agrado y autoconcepto sobre el manejo de la tecnología.

iii) Aspectos del contexto. Se dirige a obtener información del entorno físico donde se ubica el estudiante durante la consulta, además de distractores. 
iv) Aspectos del diseño instruccional. Aborda la funcionalidad, organización, claridad, diseño de contenidos, logro de aprendizaje y valoración general.

Se utilizaron 40 reactivos para conformar el cuestionario. De ellos, 27 fueron diseñados para recuperar información en escala de razón (0-10) y los 13 restantes en escala nominal dicotómica/politómica y ordinal.

Para el análisis de los datos se empleó el programa Statistical Package for the Social Sciences (SPSS), versión 22. Se aplicaron técnicas descriptivas para el análisis de los datos con apoyo de tablas y gráficos. Para los reactivos con respuestas abiertas se identificaron las ideas principales a través de categorías inductivas. Dichas categorías se establecieron a través de la revisión de la literatura acerca de aspectos específicos del diseño, como el uso de animación, legibilidad del texto, entre otras. Se buscaron los adjetivos otorgados a los aspectos técnicos de los videos, tales como agradable, confuso, atractivo, entre otros adjetivos, para agrupar y contabilizar las ocurrencias de las opiniones. Para la representación gráfica se manejaron nubes de palabras con indicadores de ocurrencias mediante el sitio web de TagCrowd (https://tagcrowd.com).

\section{Resultados}

Un punto de partida fue explorar la fiabilidad de las puntuaciones obtenidas, indicador del grado de precisión de la medida. La idea fue disponer de un instrumento que ante múltiples aplicaciones proporcionara resultados similares. Para ello, se calculó el coeficiente Alpha de Cronbach ( $\alpha$ ) para los reactivos (16-42) asociados a la evaluación de los contenidos digitales desarrollados. La fórmula aplicada corresponde a la descrita en Nunnally y Bernstein (1994):

$$
\alpha=\frac{k}{k-1}\left[1-\frac{\sum \sigma_{i}^{2}}{\sigma_{t}^{2}}\right]
$$

Donde:

k= número de ítems

$\sum \sigma_{i}^{2}=$ suma de las varianzas de los ítems

$\sigma_{t}^{2}=$ varianza de los totales 
El valor obtenido de Alpha de Cronbach para los 27 ítems fue de 0.89, lo que sugiere una adecuada fiabilidad de las puntuaciones obtenidas.

\subsection{Características del grupo}

El grupo se conformó por 40 estudiantes universitarios, 30 mujeres y 10 hombres. La media de calificaciones de los dos semestres cursados fue de 89.4 (escala 0-100). No se encontraron diferencias significativas entre las medias de calificaciones de los estudiantes hombres (88.2) y estudiantes mujeres (89.7).

Cinco enunciados del cuestionario se dirigieron a estimar aspectos de habilidad, gusto y uso educativo de la tecnología y conocimiento previo de Estadística. La Figura 5 muestra, en escala 0-1 donde 0 es nulo y 1 máximo, los valores calculados para los puntos mencionados. Se destacan los máximos obtenidos para la habilidad y gusto por la tecnología; sin embargo, se exhibe una baja percepción de dominio en los temas estadísticos.

Figura 5.

Aspectos asociados al uso de la tecnología por las personas participantes

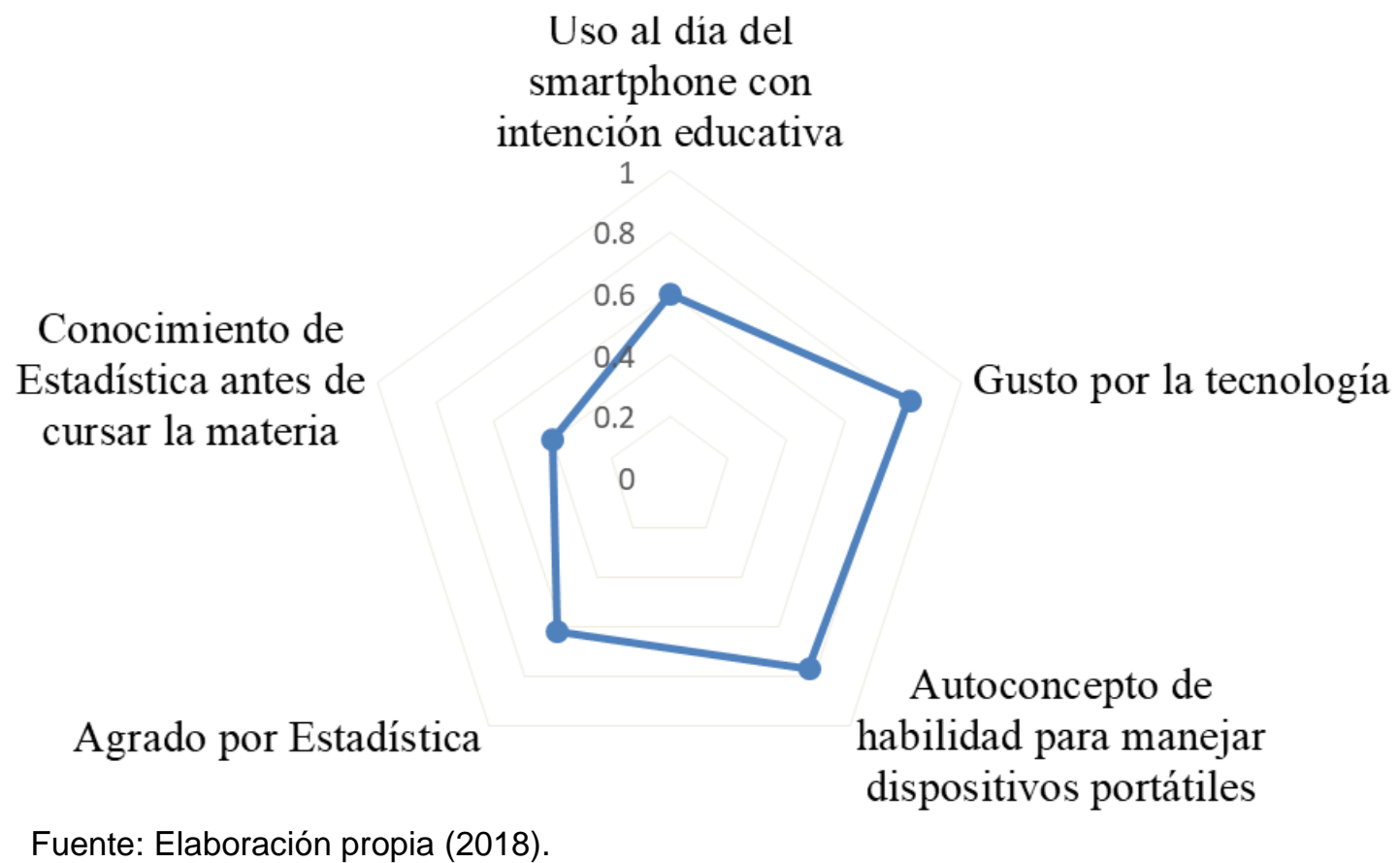




\subsection{El acceso a los contenidos educativos digitales: equipo, conexión y distractores}

El total (100\%) de los participantes dispone de algún tipo de teléfono inteligente y $83 \%$ cuenta con una laptop. La tableta es el dispositivo con menor porcentaje de posesión (28\%) en el grupo de estudio. En cuanto a la conectividad de sus dispositivos, $80 \%$ del grupo participante cuenta con algún plan de datos que le permite conexión 3G/4G a Internet, aunado a los accesos abiertos o públicos de redes $\mathrm{Wi}-\mathrm{Fi}$. Apesar de que los CED se orientaron desde un teléfono inteligente, donde el 58\% lo hizo desde este dispositivo, el porcentaje restante accedió a través de un laptop o tableta. La plataforma de distribución de los contenidos mayormente utilizada fue Google Classroom con un 95\%; sóoo 5\% recurrió a WhatsApp.

Se solicitó al grupo participante identificar los tres principales distractores durante la consulta a los contenidos educativos. Destacan las notificaciones de redes sociales (62\%) y los ruidos del exterior (47\%) como los mayormente mencionados.

Figura 6.

Principales distractores identificados por las personas participantes

Youtube
Yotificación de App
Nonto al transportarse
Meflejo de la luz en la pantalla
Músa propia
Problemas con el dispositivo
Llamada entrante
Recepción de mensajes
Ruidos del exterior

Notificación de redes sociales

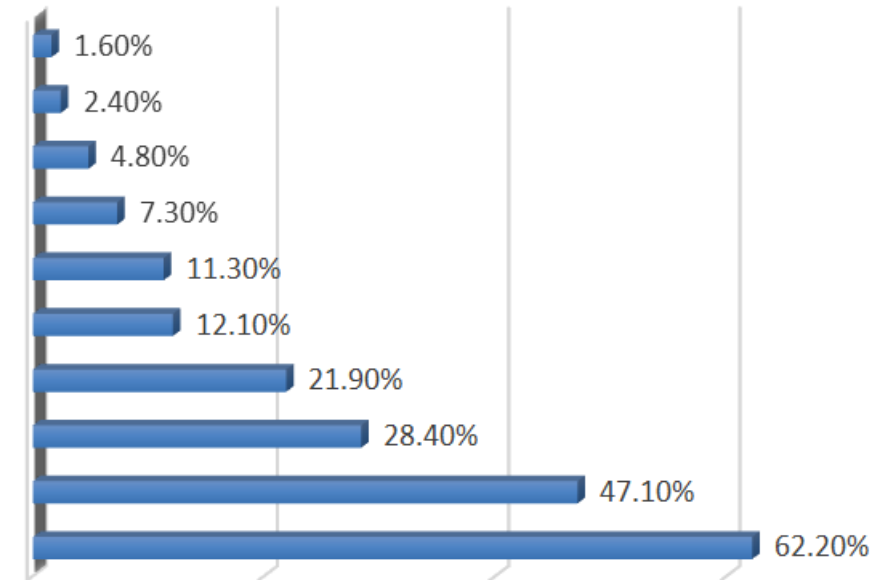

Fuente: Elaboración propia del autor (2018).

\subsection{Opinión del estudiantado acerca de los CED}

Para evaluar la opinión de quienes participaron en el diseño instruccional empleado en los CED, se analizaron 27 reactivos en escala 0-10 del cuestionario aplicado. En términos generales, la Figura 7 da cuenta de valoraciones en el rango 9-10, con excepción del aspecto de la claridad de los contenidos, tópico que comprendía enunciados en torno a la 
claridad de procedimientos, explicación de conceptos, lenguaje utilizado y velocidad de la explicación.

Figura 7.

Valoración delos aspectos del diseño instruccional utilizado por las personas participantes

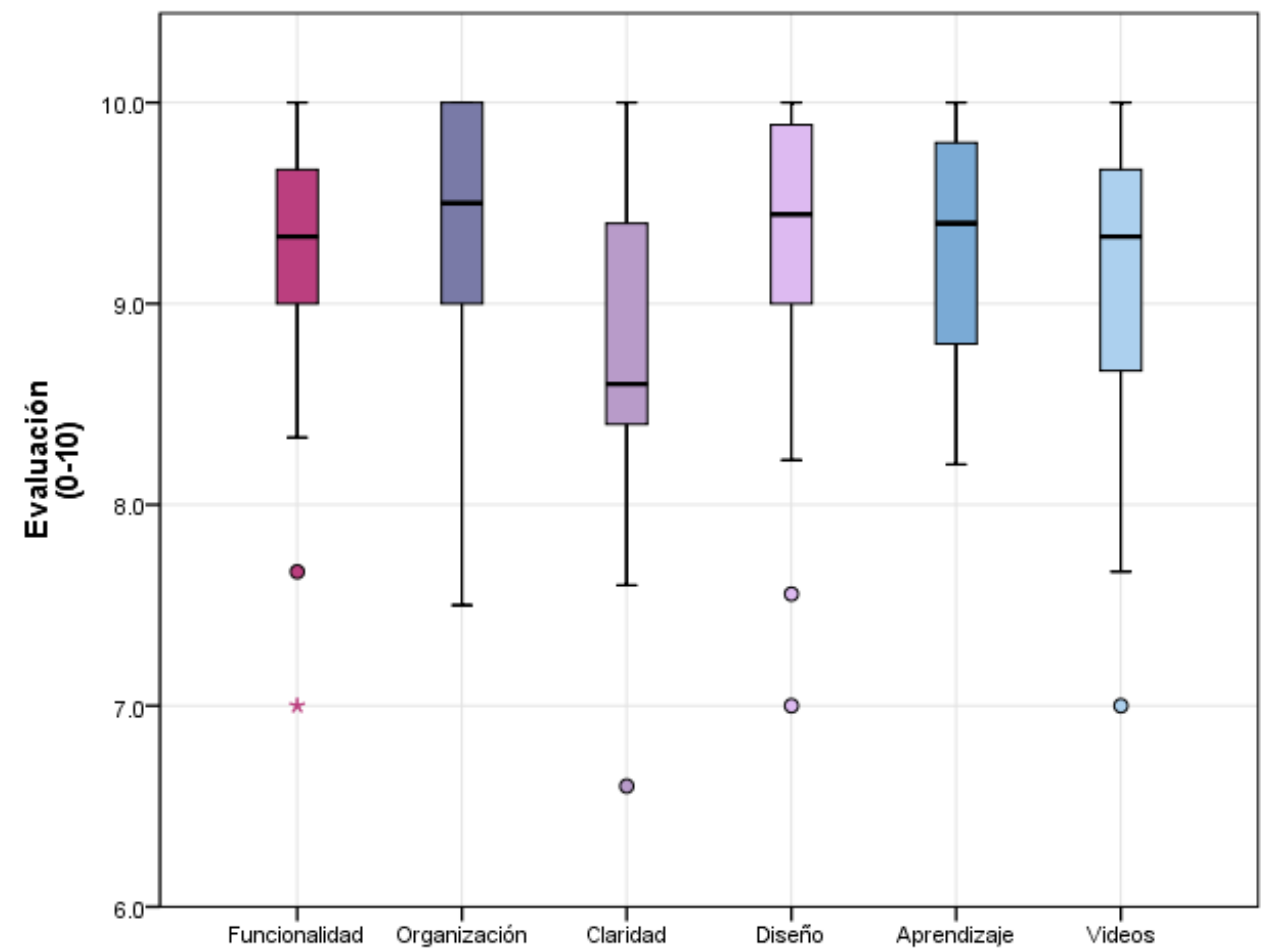

Fuente: Elaboración propia (2018).

El cuestionario aplicado incluyó, en su parte última, dos reactivos de respuesta abierta para que quienes participaron pudieran indicar lo que fue de su agrado y desagrado con respecto alos contenidos consultados. Para su análisis, se identificaron las ideas principales y se codificaron, para su representación gráfica, en nubes de palabras. La Figura 8 presenta la nube de palabras generada con la opinión de desagrados, donde se destacan los comentarios de explicaciones no detalladas o rápidas, y dudas a lo visto. 
Figura 8.

Términos de desagrado hacia los CED expresados por el grupo participante

contuso (1) Dudas

Errores Explicacion-no-

d e t a I l a d a

Explicacion-rapida

(9) Nás-ejemPlOS (2) Música-distractor (1) No-entender-resuitado (1)

No-entendio (2) Problemas-Internet

Repetir-video (2) Video-rápido (1) Visibilidad (1) Voz (1) Voz-

rápida (2)

Fuente: Elaboración propia (2018).

En cuanto a los aspectos que fueron de agrado expresados por el grupo participante, la Figura 9 destaca la explicación entendible, la posibilidad de repetir el segmento audiovisual y el acceso fácil. 
Figura 9.

Términos de desagrado hacia los CED expresados por el grupo participante

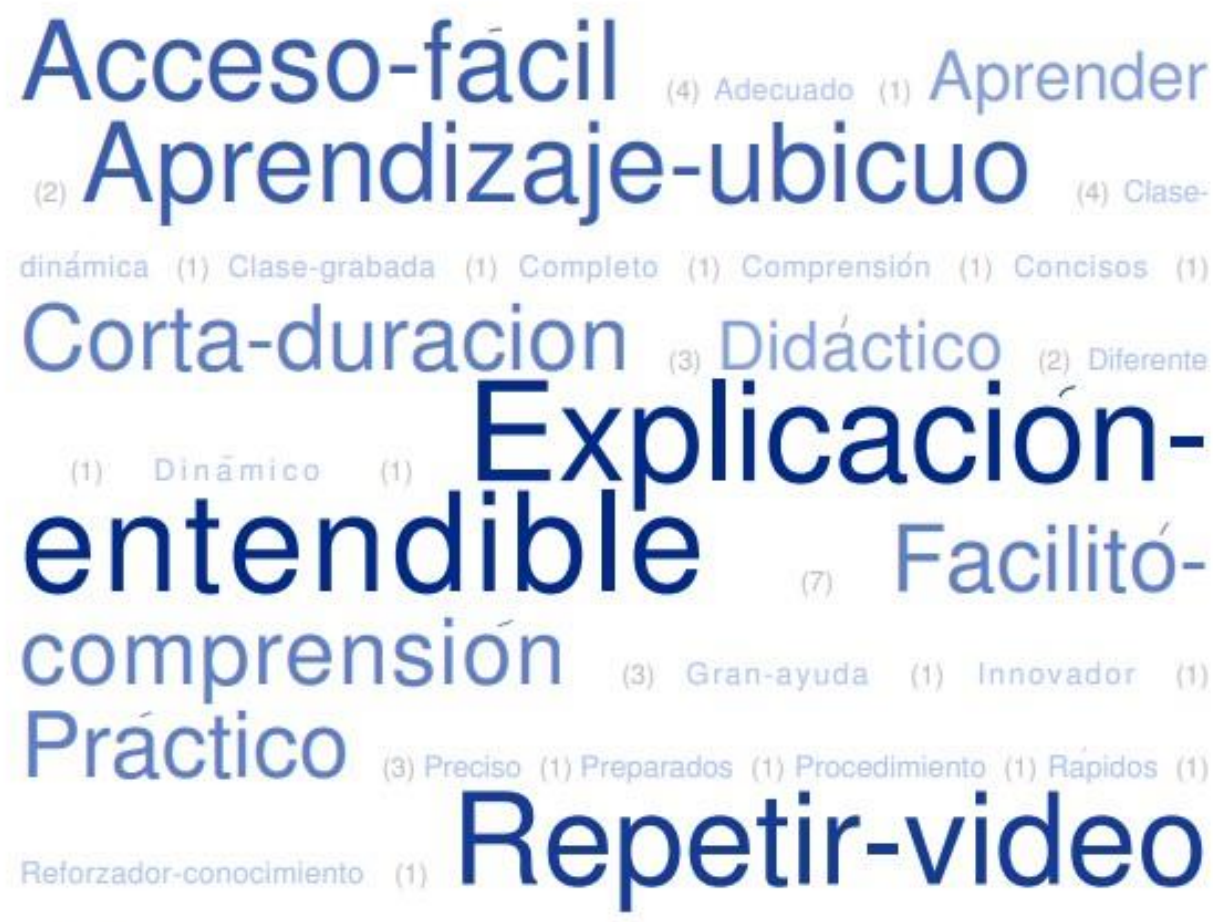

(6) Sencillos (1)

Fuente: Elaboración propia del autor (2018).

\section{Conclusiones}

Un punto de partida es destacar que el instrumento utilizado para valorar la opinión del grupo participante muestra una adecuada fiabilidad de las puntuaciones obtenidas. Ello se deriva del valor estimado de Alpha de Cronbach de 0.89. Se espera que para aplicaciones posteriores produzca resultados similares.

El grupo de estudio se caracterizó por estar conformado predominantemente por mujeres (3 de cada 4). En términos de sus medias de calificaciones, no se registraron diferencias significativas según el género. Llama la atención que el 100\% del estudiantado cuenta con un teléfono inteligente y poco más de $80 \%$ dispone de algún plan de datos para acceder a Internet. Los y las estudiantes del estudio reflejaron un perfil de agrado hacia la tecnología, habilidades para el manejo de dispositivos portátiles y un uso habitual de los servicios de Internet, especialmente para acceder a información y establecer comunicación. Las características narradas dan cuenta de condiciones tecnológicas adecuadas para 
propiciar el aprendizaje del estudiantado, pese a la baja percepción de dominio señalada para los temas estadísticos.

Para la distribución de los CED, 95\% de los estudiantes utilizaron la plataforma de Google Classroom, y solo 5\% recurrió a WhatsApp. Es importante destacar que, si bien, todos refieren contar con un teléfono inteligente, únicamente $58 \%$ consultó los contenidos desde su dispositivo. El porcentaje restante utilizó laptop o tableta. Sobre este punto se registra que $83 \%$ de los estudiantes cuentan con un teléfono inteligente y laptop, por lo que se diversifican las posibilidades de consulta a los contenidos. En consecuencia, hubo quienes señalaron que usaron la laptop para facilitar la lectura ante lo reducido de la pantalla del Smartphone.

Respecto a la valoración acerca del diseño instruccional empleado en los CED, los resultados mostraron valores medios en el rango 9-10 de la escala 0-10; en especial, el grupo participante señaló los aspectos de organización de los contenidos, el diseño empleado, su funcionalidad y su utilidad para favorecer la comprensión de los temas y favorecer su aprendizaje. Pero también destacaron lo complicado de los temas estadísticos, ya que independientemente de los apoyos tecnológicos empleados, la dificultad persiste.

En torno a los aspectos negativos de los CED utilizados, los estudiantes señalaron la falta de detalle en las explicaciones, lo rápido de los videos, o dudas a lo visto. Respecto a los principales distractores, mencionaron la insistente notificación de redes sociales, los ruidos del exterior y la recepción de mensajes. En cuanto a los aspectos positivos, destacaron que la explicación fue entendible, que se podía repetir el segmento audiovisual y la facilidad de acceso en todo momento y lugar. La experiencia presentada en el documento da cuenta del potencial pedagógico que ofrecen los teléfonos inteligentes a través de los recursos de información y comunicación disponibles en todo momento y lugar. Asimismo, estos recursos pueden utilizarse por medio de aplicaciones, hipervínculos, contenidos digitales desarrollados por programas de diseño responsivo o vía 'streaming' de segmentos audiovisuales, entre otros. Indudablemente, el uso adecuado de tales recursos tecnológicos en las actividades educativas propiciará mejores condiciones para el aprendizaje. 


\section{Referencias}

Agudelo, Mónica. (2009). Importancia del diseño instruccional en ambientes virtuales de aprendizaje. En J. Sánchez. Nuevas Ideas en Informática Educativa, 5, 118-127. Recuperado de http://www.tise.cl/volumen5/TISE2009/Documento15.pdf

Aparici, R. (mayo, 2011). Principios pedagógicos y comunicacionales de la educación 2.0. Revista Digital la educ@ción, 145. Recuperado de http://www.educoas.org/portal/La Educacion Digital/laeducacion 145/articles/Robert o Aparici.pdf

Basantes, A., Naranjo, M., Gallegos, M. y Benítez, N. (2017). Los dispositivos móviles en el proceso de aprendizaje de la facultad de educación ciencia y tecnología de la Universidad Técnica del Norte de Ecuador. Formación Universitaria. 10(2), 79-88. DOI: http://dx.doi.org/10.4067/S0718-50062017000200009

Batanero, Carmen. (2001). Didáctica de la Estadística. Granada, españa: GEEUG. Recuperado http://www.ugr.es/ batanero/pages/ARTICULOS/didacticaestadistica.pdf

Batanero, Carmen y Díaz, Carmen. (2004). El papel de los proyectos en la enseñanza y aprendizaje de la estadística. En J. Patricio Royo (Ed.), Aspectos didácticos de las matemáticas (pp. 125-164). Zaragoza, España: ICE. Recuperado de http://www.ugr.es/ batanero/pages/ARTICULOS/ICE.pdf

Berney, Sandra. y Bétrancourt, Mireille. (2016). Does animation enhance learning? A metaanalysis. Computers \& Education, 101, 150-167. doi: https://doi.org/10.1016/i.compedu.2016.06.005

Bétrancourt, Mireille. (2005). The animation and interactivity principles in multimedia learning. En R. Mayer (Ed.) The Cambridge Handbook of Multimedia Learning. California, Estados Unidos: Cambrigde University Press. 278-296. doi: https://doi.org/10.1017/CBO9780511816819.019

Chiappe. Andrés. (2008). Diseño instruccional: oficio, fase y proceso. Educación y Educadores, 11(2), 229-239. Recuperado de http://www.redalyc.org/pdf/834/83411215.pdf

Chiappe, Andrés. (2016). Tendencias sobre contenidos educativos digitales en América. Buenos Aires, Argentina: UNESCO. Recuperado de http://unesdoc.unesco.org/images/0024/002456/245673s.pdf

Denham, A., Quick, J. y Atkinson, R. (2012). MLearning: An embodied perspective. International Journal of Cyber Behavior, Psychology and Learning, 2(3), 1-

14. Recuperado de http://dx.doi.org/10.4018/ijcbpl.2012070101 
Díaz Perera, Juan José., Saucedo Fernández, Mario., Salinas Padilla, Heidi Angélica. y Jiménez Izquierdo, Sergio. (2014). Las TIC: distractores o herramientas para el aprendizaje. Revista Iberoamericana de Producción Académica y Gestión Educativa, (2).

http://www.pag.org.mx/index.php/PAG/article/view/189/237

Donkor, Francis. (2011). Assessment of learner acceptance and satisfaction with video-based instructional materials for teaching practical skills at a distance. International review of research in open and distance learning, 12(5), 74-92. Recuperado de http://www.irrodl.org/index.php/irrodl/article/view/953/1891

Enrico, R., Casanova, B., Enrico, E. y Bossolasco, M. (2017). El diseño de materiales educativos digitales. Una mirada desde los estudiantes usuarios. En IV Jornadas de TIC e Innovación en el Aula. Recuperado de http://sedici.unlp.edu.ar/bitstream/handle/10915/65555/Documento completo.pdfPDFA2u.pdf?sequence $=1$

Faustino, Arnaldo. y Pérez Luis, Sara. (2014). Utilización de las TIC en la enseñanza de la estadística en la Educación Superior angolana. Prisma social, (11), 1-31. Recuperado de http://www.redalyc.org/html/3537/353744535001/

García-Barrera, Alba. (2013). El aula inversa: cambiando la respuesta a las necesidades de los estudiantes. Revista de la Asociación de Inspectores de Educación de España, (19), 1-8. Recuperado de https://avances.adide.org/index.php/ase/article/view/118/115

Instituto Nacional de Estadística y Geografía. (2018). Estadísticas a propósito del día mundial de Internet (17 de mayo). Recuperado de http://www.inegi.org.mx/saladeprensa/aproposito/2018/internet2018 Nal.pdf

Jeong, S. y Cha, C. (2017). Developing a customized sexually transmitted infections (STIs) application for adolescents: An application of the instructional system design model. Journal of the Korea Academia- Industrial, 2(18), 651-659. DOI: https://doi.org/10.5762/KAIS.2017.18.2.651

Lagunes-Domínguez, A., Torres-Gastelú, C.A., Angulo-Armenta, J. y Martínez-Olea, M.A. (2017). Prospectiva hacia el aprendizaje móvil en estudiantes universitarios. Formación Universitaria, 10(1), 101-108.

Landín Miranda, María del Rosario, Francisco Cruz, Jeannett Lizbeth y Santiago Cruz, Saraí Alberta. (2015). Internet como distractor en la vida de los estudiantes. En Ramírez Martinell, Alberto. y Casillas, Miguel Angel. (Eds.), Internet en educación superior (pp. 157-173). Córdoba. Argentina: Brujas. 
Lowe, R. (2003). Animation and learning: selective processing of information in dynamic graphics. Learning and Instruction, 13, 157-176. doi: http://dx.doi.org/10.1016/S0959$\underline{4752(02) 00018-X}$

Mancinas González, Abelardo. (2018). Modalidades de cognición en un curso universitario basado en el aprendizaje móvil. Apertura, 10(1), 40-55. Recuperado de http://dx.doi.org/10.18381/Ap.v10n1.1159

Martínez, M. y Chávez, D. (2015). Plataforma tecnológica construcción de contenidos digitales para posgrado virtual. Ra Ximhai, 11(4). Recuperado de http://www.redalyc.org/html/461/46142596027/

Martínez-Olvera, Waltraud., Esquivel-Gámez, Ismael. y Martínez-Castillo, Jaime. (2014). Aula Invertida o Modelo Invertido de Aprendizaje: Origen, Sustento e Implicaciones. En I. Esquivel, Los Modelos Tecno- Educativos, revolucionando el aprendizaje del siglo XXI (pp. 143-160). México.

Mayer, Richard. (2014). Research-based principles for designing multimedia instruction. En V. Benassi, C. Overson y C. Hakala. Applying Science of Learning in Education. (pp. 59- 70). Recuperado de http://psych.wustl.edu/memory/Roddy\%20article\%20PDF's/Pyc etal 2014 APA.pdf\# page $=64$

Nunnally, J. C. \& Bernstein, I. H. (1994). Psychometric Theory (3rd Ed.). New York: McGrawHill.

Organista-Sandoval, Javier., Sandoval Silva, Maribel., McAnally Salas, Lewis. y Lavigne, Gilles. (2016). Estimación de las habilidades digitales con propósito educativo de estudiantes de dos universidades públicas mexicanas. Revista Electrónica de Tecnología Educativa, 57. http://dx.doi.org/10.21556/edutec.2016.57.673

Organista-Sandoval, Javier. y Serrano-Santoyo, Arturo. (2014). Aspectos de posesión, permisos y usos educativos de dispositivos portátiles durante el trayecto de primaria a universidad. Apertura, 6(2). Recuperado de http://www.udgvirtual.udg.mx/apertura/index.php/apertura/article/view/570

Otero, E. A.D. (2014). Modelo de aprendizaje móvil abierto para educación superior. (Tesis de doctorado). Universidad Veracruzana, Xalapa, Veracruz, México.

Rosero, H. (2015). Aprendiendo fuera de las aulas: metodología para la construcción de un recurso educativo móvil. (Tesis de Maestría). Universidad Internacional de la Rioja, La Rioja España. Recuperado de https://reunir.unir.net/bitstream/handle/123456789/3068/HectorMiguel Rosero Florez. pdf? sequence $=1$ \&isAllowed $=y$ 
Velarde, A., Dehesa, J., López, E. y Márquez, J. (2017). Los vídeo tutoriales como apoyo al proceso de enseñanza aprendizaje y sus implicaciones pedagógicas en el diseño instruccional. Revista EDUCATECONCIENCIA, 14(15). 67-86. Recuperado de http://tecnocientifica.com.mx/educateconciencia/index.php/revistaeducate/article/view/ $\underline{330 / 307}$

Zapata Cardona, Lucía. (2011). ¿Cómo contribuir a la alfabetización estadística? Revista Virtual Universidad Católica del Norte, 33, 234-247. Recuperado de http://revistavirtual.ucn.edu.co/index.php/RevistaUCN/article/view/4/8 